\title{
Atrial fibrillation after esophagectomy is a marker for postoperative morbidity and mortality
}

\author{
Sudish C. Murthy, MD, PhD ${ }^{\mathrm{b}}$ \\ Simon Law, MBBChir, MS, FRCSEd, FACS ${ }^{a}$ \\ Brian P. Whooley, MD \\ Andreas Alexandrou, $\mathrm{MD}^{\mathrm{a}}$ \\ Kent-Man Chu, MBBS, MS, FRCSEd, FACS ${ }^{\mathrm{a}}$ \\ John Wong, PhD, FRACS, FACS ${ }^{\mathrm{a}}$
}

From the Division of Esophageal Surgery, Department of Surgery, ${ }^{\mathrm{a}}$ University of Hong Kong Medical Centre, Queen Mary Hospital, Hong Kong, China, and the Department of Thoracic and Cardiovascular Surgery, ${ }^{\mathrm{b}}$ The Cleveland Clinic Foundation, Cleveland, Ohio

S.C.M. was supported in part by a WarrenWhitman-Richardson Fellowship from Harvard Medical School and travel grant from the Division of Thoracic Surgery, Brigham and Women's Hospital, Boston, Mass.

Received for publication Dec 7, 2001; revisions requested April 23, 2002; revisions received Aug 22, 2002; accepted for publication June 22, 2003.

Address for reprints: John Wong, $\mathrm{PhD}$, Department of Surgery, University of Hong Kong Medical Centre, Queen Mary Hospital, Hong Kong, China (E-mail: jwong@hku.hk).

J Thorac Cardiovasc Surg 2003;126:1162-7

Copyright (C) 2003 by The American Association for Thoracic Surgery

$0022-5223 / 2003 \$ 30.00+0$

doi:10.1016/S0022-5223(03)00974-7
Objective: Postoperative atrial fibrillation complicates recovery in $20 \%$ to $25 \%$ of patients after esophagectomy for cancer. The purpose of this study is to understand this phenomenon.

Methods: Between 1982 and 2000, 198 (22\% of 921) patients had postoperative atrial fibrillation after esophagectomy. Propensity scoring and the Greedy Match algorithm were used to develop a cohort of control patients for statistical comparisons. One hundred forty-four patients who had postoperative atrial fibrillation were matched.

Results: Pulmonary complications affected $42 \%$ of patients in the atrial fibrillation group compared with $17 \%$ in the control group $(P<.001)$. Anastomotic leakage was more common in the atrial fibrillation group $(6.9 \%$ vs $1.4 \%, P=.035)$. Surgical sepsis migrated with atrial fibrillation 4 times more frequently $(P=.001)$. Multivariate analysis demonstrated that postoperative pulmonary complications (odds ratio, 2.5; 95\% confidence interval, 1.42-4.3) and surgical sepsis (odds ratio, 3.4; $95 \%$ confidence interval, 1.2-9.6) were associated with postoperative atrial fibrillation. The mortality rates of the atrial fibrillation and control groups were $23 \%$ and $6.3 \%$, respectively $(P<.001)$. Median survival, excluding hospital deaths, was not different at 14.5 months (atrial fibrillation group) and 16.9 months (control group; $P=.4)$.

Conclusion: Atrial fibrillation is a surrogate for surgical morbidity and mortality after esophagectomy. The occurrence of atrial fibrillation after esophageal resection should prompt not only the appropriate management of the arrhythmia but also a search for a more ominous underlying cause.

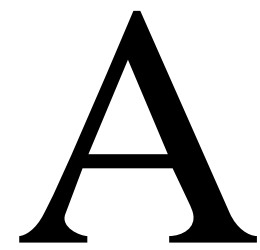

trial fibrillation (AF) is the most common sustained cardiac arrhythmia. ${ }^{1} \mathrm{AF}$ after coronary artery bypass graft surgery is generally considered a self-limiting and benign process, with thromboembolism as a major sequela. ${ }^{2}$ Atrial tachyarrhythmia is also a well-recognized complication of noncardiac thoracic surgery. The reported incidence of $\mathrm{AF}$ complicating recovery from thoracic operations approaches $30 \%$ in some series. ${ }^{3-7}$ Unfortunately, unlike coronary surgery, the development of AF after noncardiac thoracic surgery, specifically lung surgery, is associated with a worse outcome..$^{3,4,6,8}$ The cause of AF after lung surgery is unclear, although some have cited arterial hypertension, ischemic heart disease, ${ }^{8,9}$ and pulmonary malignancy ${ }^{5}$ as preoperative risk factors. Recent studies have demonstrated pneumonectomy (compared with lesser resections) and age as risks for AF. ${ }^{10,11}$ 
Surgical resection for carcinoma of the esophagus and gastric cardia is arguably one of the most morbid thoracic operations. ${ }^{12}$ Although patient selection and improved postoperative care can significantly decrease hospital mortality, ${ }^{13-15}$ morbidity remains high. ${ }^{12,13,16} \mathrm{AF}$ frequently complicates recovery from esophagectomy, ${ }^{17,18}$ and its significance remains unclear. In this study risk factors for the development of postoperative AF were identified. Moreover, the effect of AF on postoperative outcome was examined.

\section{Methods}

Between July 1982 and December 2000, 1816 patients with cancer of the esophagus or gastric cardia were managed at the University of Hong Kong Medical Center, Queen Mary Hospital. Of these patients, 1138 underwent surgical resection. Patients who underwent pharyngolaryngoesophagectomy, patients with gastric cardia cancer and an abdominal resection, and patients with staged resections were excluded. Prospectively collected data from the remaining 921 patients were retrospectively reviewed.

Preoperative assessment of all patients included a thorough medical history and complete physical examination, standard blood chemistries and blood counts, and cardiopulmonary evaluation. A history of cardiac disease included at least one of the following: hypertension, atrial or ventricular arrhythmia, ischemic heart disease, congestive heart failure, or valvular disease. A history of pulmonary disease included emphysema, bronchiectasis, or tuberculosis.

All patients had a barium contrast study, flexible fiberoptic upper endoscopy, and bronchoscopy. Computed tomographic scans and endoscopic ultrasonography were also routinely performed in recent years. The operative approach was individualized for each patient and was governed by the location and stage of the tumor, the history of previous abdominal surgery, intraoperative findings, and preoperative comorbid disease. Specific surgical techniques have been described previously. ${ }^{19,20}$ Patients with cardia cancer were resected through an abdominal-right thoracic approach (sixth or seventh intercostal space). In these patients the esophagogastric anastomosis was below the level of the carina, and consequently, the extent of intrathoracic dissection was restricted. The operation was considered curative when the tumor was completely resected with microscopically negative margins.

\section{Outcomes}

AF was documented by means of electrocardiography, with the majority of patients requiring medical intervention for rate control, rhythm control, or both. Treatment included cardioversion, digoxin, calcium channel or $\beta$-blockade, and intravenous amiodarone.

Study end points included several possible postoperative outcomes. Cardiovascular complications were ventricular arrhythmia, congestive heart failure and circulatory overload, myocardial infarction, and pulmonary embolus. Aspiration pneumonia, bronchopneumonia, pulmonary edema, respiratory failure, and shock lung were included as pulmonary complications. Nonanastomotic leakage occurred from the lesser curve gastric staple line, ischemic necrosis of the esophageal substitute, or any other nonanastomotic site and was distinguishable from anastomotic leak in all cases. Endoscopy, barium contrast study, or computed tomographic scans were used to document leak. Sepsis was defined as hemodynamic instability and end-organ (pulmonary, renal, hepatic, and central nervous system) dysfunction. The cause of sepsis was both medical (eg, pneumonia) and surgical (eg, leak related). Unless otherwise specified, mortality was until the day of discharge (hospital mortality). Thirty-day mortality was also recorded for all patients.

\section{Statistical Method and Generation of the Control Group}

Propensity scoring and the Greedy Match algorithm ${ }^{21-24}$ were used to develop a control population suitable for statistical comparison. Twenty-one factors were analyzed by using multivariate logistic regression to identify variables that correlated with postoperative AF (Appendix 1). The 4 variables found to be predictive of postoperative AF were age $(P<.0001)$, history of cardiac disease $(P=.007)$, amount of blood loss $(P=.015)$, and limited intrathoracic dissection $(P=.002)$. These 4 factors were then used to generate a matched control group by using the Greedy Match algorithm. In brief, a propensity score for development of postoperative $\mathrm{AF}$ was generated for each patient in the entire population by means of logistic modeling with the 4 variables identified above. Patients with postoperative AF were matched to patients without AF on the basis of propensity score. The $\mathrm{C}$ statistic was 0.7 , and the Hosmer-Lemeshow goodness-of-fit test had a $P$ value of .1. One hundred forty-four patients with postoperative AF (study group) were matched with 144 patients who did not have $\mathrm{AF}$ (control group) by means of this methodology. The remaining 54 unmatched patients with postoperative AF were compared with the matched patients with AF (Appendix 2). Unmatched patients had a higher frequency of preoperative pulmonary disease and a higher preoperative serum hemoglobin level. Transhiatal esophagectomy was more prevalent in the unmatched group.

All continuous variables are expressed as means \pm SD unless otherwise stated. Univariate analyses were performed by means of analysis of variance for continuous variables and $\chi^{2}$ testing for categoric variables. The Fisher exact test was used if any expected cell value in a $2 \times 2$ table was less than 5 . Stepwise logistic regression analysis was used for multivariate analysis. Survival curves were generated by means of Kaplan-Meier methodology. All calculations were conducted with biostatistical programs of SPSS (Chicago, Ill) and Statistical Analysis System software (version 6.12; SAS, Cary, NC).

\section{Results}

Of the 921 patients in the population, 198 (22\%) who had postoperative AF were identified. Appropriate control subjects were identified for 144 patients with AF. The preoperative demographics of the matched groups are shown in Table 1. Operative factors are shown in Table 2. These data demonstrate that the 2 groups have been successfully matched on the basis of the methodology applied. Notably, significant variables identified in the initial analysis (age, cardiac disease, blood loss, and limited thoracic dissection) were well matched after propensity scoring and application of the Greedy Match algorithm. 
TABLE 1. Demographics of patients with postoperative atrial arrhythmias (AF group) compared with those of patients without AF (control group)

\begin{tabular}{lccc}
\hline & AF group & Control group & $\boldsymbol{P}$ value \\
\hline No. & 144 & 144 & - \\
Age $(y \pm$ SD) & $66.8 \pm 8.4$ & $67 \pm 8.3$ & .83 \\
Male/female sex & $116: 28$ & $119: 25$ & .76 \\
Smoking history & 108 & 103 & .6 \\
Histology of tumor & & & \\
$\quad$ Squamous & 128 & 133 & .5 \\
$\quad$ Adenocarcinoma & 13 & 10 & \\
$\quad$ Others & 3 & 1 & \\
Level of tumor & & & \\
$\quad$ Upper 1/3 & 9 & 11 & .7 \\
$\quad$ Middle 1/3 & 91 & 91 & \\
$\quad$ Lower 1/3 & 30 & 33 & \\
$\quad$ Cardia & 14 & 9 & \\
Pulmonary disease & 17 & 21 & .6 \\
Cardiac disease & 31 & 32 & 1.0 \\
Albumin (g/dL \pm SD) & $41 \pm 4.2$ & $41 \pm 4.5$ & .49 \\
Hemoglobin (g/dL \pm SD) & $12.6 \pm 1.8$ & $13 \pm 1.5$ & .49 \\
\hline
\end{tabular}

Figures represent numbers of patients unless stated otherwise.

TABLE 2. Intraoperative factors in patients with atrial arrhythmias (AF group) compared with those in patients without AF (control group)

\begin{tabular}{lccc}
\hline & AF group & Control group & P value \\
\hline No. & 144 & 144 & \\
Surgical procedures & & & \\
$\quad$ Lewis Tanner & 80 & 82 & .7 \\
$\quad$ Transhiatal & 18 & 23 & \\
$\quad$ Three-phase & 25 & 17 & \\
$\quad$ Esophagogastrectomy & 16 & 17 & \\
$\quad$ Thoracoscopy & 5 & 5 & \\
Thoracotomy & & & \\
$\quad$ Yes & 127 & 118 & .19 \\
$\quad$ No & 17 & 26 & \\
Mediastinal dissection above T5 & & & \\
Yes & 130 & 131 & 1.0 \\
$\quad$ No & 14 & 13 & \\
Intent of operation & & & \\
$\quad$ Curative & 66 & 63 & .8 \\
$\quad$ Palliative & 78 & 81 & \\
Blood loss (mL \pm SD) & $845 \pm 583$ & $798 \pm 711$ & .54 \\
Operative time (min \pm SD) & $240 \pm 66$ & $235 \pm 51$ & .5 \\
Stage of disease & & & \\
$\quad$ I & 21 & 24 & .79 \\
Ila & 35 & 33 & \\
$\quad$ Ilb & 7 & 6 & \\
III & 73 & 77 & \\
IV & 8 & 4 &
\end{tabular}

Figures represent numbers of patients unless stated otherwise.

The creation of the control group enabled meaningful analysis of the effect of postoperative AF on the outcome of patients. The differences in postoperative complications be-
TABLE 3. Postoperative complications in patients with AF compared with those in patients without AF (control group)

\begin{tabular}{lccc}
\hline & AF group & Control group & $\boldsymbol{P}$ value \\
\hline No. & 144 & 144 & \\
Medical & & & \\
$\quad$ Cardiac* & 16 & 7 & .08 \\
Pulmonary & 61 & 25 & $<.001$ \\
Hepatic failure & 2 & 1 & .1 \\
Renal failure & 8 & 0 & .01 \\
$\quad$ Stroke & 1 & 0 & 1.0 \\
Surgical & & & \\
$\quad$ Anastomotic leak & 10 & 2 & .035 \\
Nonanastomotic leak & 8 & 2 & .05 \\
All leaks & 18 & 3 & .001 \\
Surgical sepsis & 22 & 5 & .001 \\
Wound complications & 23 & 14 & .16 \\
\hline
\end{tabular}

Figures represent numbers of patients.

*Cardiac complications excluding atrial arrhythmia.

tween the 2 groups are shown in Table 3 . Significantly more patients in the AF group had pulmonary complications. The severity of pulmonary morbidity is demonstrated by the 2.4-fold greater incidence of tracheostomy required in the postoperative AF group (64 patients vs 26 patients, $P<$ .001). Renal failure was also more common in the AF group. A 6-fold difference in the absolute leak rate between populations was observed. This was likely manifested by the high incidence of surgical sepsis complicating patients in the AF group (22 patients vs 5 patients, $P<.001$ ).

Multivariate analysis identified postoperative pulmonary complications (odds ratio, 2.5; 95\% confidence interval, 1.42-4.3) and surgical sepsis (odds ratio, 3.4; 95\% confidence interval, 1.2-9.6) as independent variables associated with postoperative AF after esophagectomy. Seventy (49\%) patients with postoperative AF had at least one of these complications.

A striking disparity existed between hospital mortality rates of patients with and without AF (Figure 1). Overall, the mortality was 3.7 times greater when AF was observed during the postoperative course $(P<.001)$. Although the ability to recover patients from esophagectomy improved with time, postoperative AF continued to correlate with mortality regardless of early (1982-1990) or recent experience (1991-2000). The median survival was shorter for patients with postoperative AF compared with control subjects (11.5 months vs 14.5 months, $P=.02)$. However, when hospital mortality was excluded from the analysis, survival was not different (14.5 months vs 16.9 months, $P=$ $.4)$.

\section{Discussion}

The aim of this study was to understand the significance of AF after esophagectomy. Our results indicate that although 
often considered an indolent nuisance, the onset of AF after esophagectomy portends a much worse than expected surgical outcome. Specifically, we have demonstrated an increased incidence of major pulmonary complications and surgical sepsis, as well as a startling mortality rate, in patients with AF after esophageal resection. To establish these associations, we used propensity scoring and the Greedy Match algorithm to develop an appropriate control population for statistical comparisons. This methodology allows for nonrandomized comparisons to be made.

Our preliminary analysis demonstrated that advancing age and a history of cardiac disease predisposed to postoperative AF. This is not surprising because similar associations have been demonstrated for pulmonary ${ }^{8-11}$ and cardi$\mathrm{ac}^{25,26}$ surgery. Hypertension has also been shown to be a risk factor for $\mathrm{AF}$ after cancer surgery. ${ }^{27}$

Intraoperative blood loss and extensive thoracic dissection (required for a high-chest or cervical anastomosis) also migrated with postoperative AF. From previous work, we found no appreciable difference in the incidence of postoperative AF when transhiatal and Lewis-Tanner techniques were contrasted. ${ }^{17,18}$ However, included in the current study was a cohort of patients whose thoracic dissection was restricted below the level of the carina and anastomosis placed in the low chest. Most of these patients had cardia cancer, and this more limited resection does not appear to compromise tumor clearance. ${ }^{28}$ It is unclear as to why this lesser resection is associated with a lower incidence of postoperative AF. Clearly, less of the posterior mediastinum is dissected, and perhaps less single-lung ventilation time is required compared with the Lewis-Tanner approach. In addition, vagal nerves cephalad to the inferior pulmonary veins are left largely unperturbed in these resections, and this might be important. Ultimately, preservation of lung function might be the most important beneficiary of the limited thoracic dissection. Regardless, this should be considered when planning the surgical approach for cardia cancers. It would be interesting to evaluate the incidence of AF after video-assisted esophageal resections. ${ }^{29}$ For lung resections, video-assisted procedures boast similar rates of postoperative AF compared with those of open resections. ${ }^{30}$

Major pulmonary complications and surgical sepsis were frequent morbidities in patients with $\mathrm{AF}$, as documented by means of multivariate analysis. We suspect that in such patients postoperative AF is merely a systemic manifestation of these serious illnesses, and in almost $50 \%$ of patients, the onset of AF should be considered a surrogate for these grave complications. We attempted to study the temporal relationship between the development of $\mathrm{AF}$ and the diagnosis of either morbidity. Unfortunately, we had insufficient data on many of the early patients in the study to properly address this query. The results of a limited review

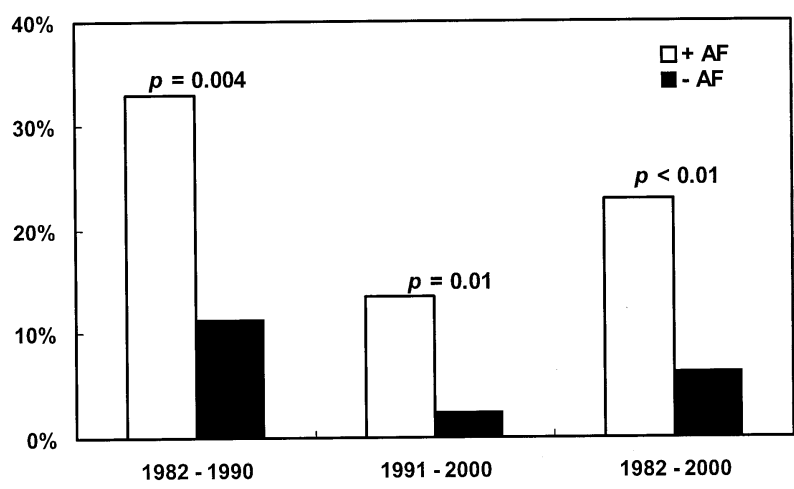

Figure 1. Hospital mortality rates after esophagectomy of patients with and without postoperative AF. Although there is a decreased mortality rate during the most recent experience, AF remains associated with poor postoperative outcome throughout the study period.

of patients who had both AF and surgical sepsis after esophagectomy suggested that when AF was noted between 3 and 10 days postoperatively, it heralded the onset of sepsis by 1 day. AF occurring at 0 to 3 days after resection demonstrated no temporal connection to the diagnosis of sepsis (unpublished data). If true, this might define a group of patients whose AF is self-limiting and attributable to the trauma and electrolyte imbalances that routinely accompany esophagectomy.

An alarming mortality rate was observed for patients who had AF after esophagectomy $(>20 \%)$. The association of postoperative AF with mortality has also been documented after lung surgery ${ }^{3,4,6,8}$ and, more recently, after major noncardiac and nonthoracic operations. ${ }^{31}$ Although improvements in intensive care and refinement of surgical techniques have decreased the postoperative death rate after esophagectomy, ${ }^{32}$ the striking association of AF with hospital mortality remained throughout the study period.

In summary, AF after esophageal resection is a marker for poor short-term outcome and frequently acts as a surrogate for pulmonary complications and surgical sepsis. In this regard strategies designed to prevent postoperative $\mathrm{AF}^{33}$ should be met with tempered optimism because we found that postoperative AF is not particularly difficult to manage. A controlled trial of prophylaxis against postoperative AF might be necessary to help validate this contention. That AF after esophagectomy is accompanied by a 6-fold increased leak rate, as well as the serious morbidities described above, should alert the surgeon to not simply treat the rhythm disturbance but to more thoroughly evaluate the postoperative patient with new-onset AF. This strategy might identify an underlying life-threatening but potentially treatable complication. 


\section{Appendix 1. List of variables used for multivariate linear regression to define elements used in the Greedy Match algorithm}

\begin{tabular}{|c|c|}
\hline Variables & $P$ value \\
\hline Date of operation & - \\
\hline Age & $<.0001$ \\
\hline Sex & - \\
\hline Histology of tumor & - \\
\hline Location of tumor & - \\
\hline Stage of disease & - \\
\hline \multicolumn{2}{|l|}{ Preoperative } \\
\hline Cardiac disease & .007 \\
\hline Pulmonary disease & - \\
\hline Serum albumin & - \\
\hline Hemoglobin & - \\
\hline Smoking history & - \\
\hline $\mathrm{FEV}_{1}$ & - \\
\hline FVC & - \\
\hline $\mathrm{Po}_{2}$ & - \\
\hline $\mathrm{PCO}_{2}$ & - \\
\hline \multicolumn{2}{|l|}{ Intraoperative } \\
\hline Thoracotomy & - \\
\hline Extent of thoracic dissection & .002 \\
\hline Intent of resection & - \\
\hline Estimated blood loss & .015 \\
\hline Length of operation & - \\
\hline
\end{tabular}

$F E V_{1}$, Forced expiratory volume in 1 second; $F V C$, forced vital capacity.

Appendix 2. Analysis of matched and unmatched patients with postoperative AF

\begin{tabular}{lccc}
\hline Variable & $\begin{array}{c}\text { Matched } \\
\text { patients } \\
(\mathbf{n}=\mathbf{1 4 4})\end{array}$ & $\begin{array}{c}\text { Unmatched } \\
\text { patients } \\
(\mathbf{n}=\mathbf{5 4 )}\end{array}$ & $\boldsymbol{P}$ value \\
\hline Male sex & 116 & 47 & .2 \\
Smoking history & 108 & 46 & .09 \\
Squamous cancer & 128 & 48 & .4 \\
History of pulmonary disease & 27 & 20 & .007 \\
History of cardiac disease & 31 & 14 & .6 \\
FEV (\% predicted) $_{\text {Preoperative albumin (g/L) }}$ & 91 & 90 & .7 \\
Preoperative hemoglobin (g/dL) & 10.5 & 40 & .7 \\
Thoracotomy & 12.6 & 13.2 & .05 \\
& 127 & 40 & .02
\end{tabular}

Figures represent numbers of patients unless otherwise stated. FEV Forced expiratory flow in 1 second.

\section{References}

1. Narayan SM, Cain ME, Smith JM. Atrial fibrillation. Lancet. 1997; 350(9082):943-50.

2. Mathew JP, Parks R, Savino JS, Friedman AS, Koch C, Mangano DT, et al. Atrial fibrillation following coronary artery bypass graft surgery: predictors, outcomes, and resource utilization. MultiCenter Study of Perioperative Ischemia Research Group. JAMA. 1996;276(4):300-6.

3. Bailey C, Betts R. Cardia arrhythmias following pneumonectomy. N Engl J Med. 1943;229:356-9.

4. Currens J, White P, Churchill E. Cardiac arrhythmias following thoracic surgery. $N$ Engl J Med. 1943;229:360-4.
5. Beck-Nielsen J, Raehbeck-Soresen H, Alstrup P. Atrial fibrillation following thoracotomy for non-cardiac diseases, in particular cancer of the lung. Acta Med Scand. 1973;193:425-9.

6. Krowka MJ, Pairolero PC, Trastek VF, Payne WS, Bernatz PE. Cardiac dysrhythmia following pneumonectomy. Clinical correlates and prognostic significance. Chest. 1987;91(4):490-5.

7. Wahi R, McMurtrey MJ, DeCaro LF, Mountain CF, Ali MK, Smith TL, et al. Determinants of perioperative morbidity and mortality after pneumonectomy. Ann Thorac Surg. 1989;48(1):33-7.

8. von Knorring J, Lepantalo M, Lindgren L, Lindfors O. Cardiac arrhythmias and myocardial ischemia after thoracotomy for lung cancer. Ann Thorac Surg. 1992;53(4):642-7.

9. Dyszkiewicz W, Skrzypczak M. Atrial fibrillation after surgery of the lung: clinical analysis of risk factors. Eur J Cardiothorac Surg. 1998; 13:625-8.

10. Curtis JJ, Parker BM, McKenney CA, Wagner-Mann CC, Walls JT, Demmy TL, et al. Incidence and predictors of supraventricular dysrhythmias after pulmonary resection. Ann Thorac Surg. 1998;66:1766-71.

11. Rena O, Papalia E, Oliaro A, Casadio C, Ruffini E, Filosso PL, et al. Supraventricular arrhythmias after resection surgery of the lung. Eur J Cardiothorac Surg. 2001;20:688-93.

12. Earlam R, Cunha-Melo JR. Oesophageal squamous cell carcinoma. Br J Surg. 1980;67:457-61.

13. Bartels H, Stein HJ, Siewert JR. Preoperative risk analysis and postoperative mortality of oesophagectomy for resectable oesophageal cancer. Br J Surg. 1998;85(6):840-4.

14. Law SYK, Wong J. Complications: prevention and management. In: Daly JM, Hennessy TPJ, Reynolds JV, editors. Management of upper gastrointestinal cancer. London: W.B. Saunders; 1999. p. 240-62.

15. Law SY, Fok M, Wong J. Risk analysis in resection of squamous cell carcinoma of the esophagus. World J Surg. 1994;18(3):339-46.

16. Poon RT, Law SY, Chu KM, Branicki FJ, Wong J. Esophagectomy for carcinoma of the esophagus in the elderly: results of current surgical management. Ann Surg. 1998;227(3):357-64.

17. Chu KM, Law SY, Fok M, Wong J. A prospective randomized comparison of transhiatal and transthoracic resection for lower-third esophageal carcinoma. Am J Surg. 1997;174(3):320-4.

18. Fok M, Wong J. Cancer of the oesophagus and gastric cardia. Standard oesophagectomy and anastomotic technique. Ann Chir Gynaecol. 1995;84(2):179-83.

19. Law S, Wong J. Esophagogastrectomy for carcinoma of the esophagus and cardia, and the esophageal anastomosis. In: Baker RJ, Fischer JE, editors. Mastery of surgery. Philadelphia: Lippincott Williams \& Wilkins; 2001. p. 813-27.

20. Law S, Wong J. Esophagectomy without thoracotomy. In: Baker RJ, Fischer JE, editors. Mastery of surgery. Philadelphia: Lippincott Williams \& Wilkins; 2001. p. 828-36.

21. Parsons L. Using SAS software to perform a case-controlled method for bias reduction in an observational study. In: Proceedings of the Twenty-Fifth Annual SAS Users Group International Conference. Cary, NC: SAS Institute, Inc; 2000. p. 1166-71.

22. Blackstone EH. Comparing apples and oranges. J Thorac Cardiovasc Surg. 2002;123:8-15.

23. Rosenbaum PR, Rubin DB. Reducing bias in observational studies using subclassification on the propensity score. J Am Stat Assoc. 1984;79:516-24.

24. Rosenbaum PR. From association to causation in observational studies: the role of tests of strongly ignorable treatment assignment. $J \mathrm{Am}$ Stat Assoc. 1984;79:41-8.

25. Yousif H, Davies G, Oakley CM. Peri-operative supraventricular arrhythmias in coronary bypass surgery. Int J Cardiol. 1990;26(3):313-8.

26. Crosby LH, Pifalo WB, Woll KR, Burkholder JA. Risk factors for atrial fibrillation after coronary artery bypass grafting. Am J Cardiol. 1990;66(20):1520-2.

27. Gibbs HR, Swafford J, Nguyen HD, Ewer MS, Ali MK. Postoperative atrial fibrillation in cancer surgery: preoperative risks and clinical outcome. J Surg Oncol. 1992;50(4):224-7.

28. Siewert JR, Stein HJ, Sendler A, Fink U. Surgical resection for cancer of the cardia. Semin Surg Oncol. 1999;17:125-31.

29. Law S, Fok M, Chu KM, Wong J. Thoracoscopic esophagectomy for esophageal cancer. Surgery. 1997;122(1):8-14. 
30. Neustein SM, Kahn P, Krellenstein DJ, Cohen E. Incidence of arrhythmias after thoracic surgery: thoracotomy versus video-assisted thoracoscopy. J Cardiothorac Vasc Anesth. 1998;12(6):659-61.

31. Brathwaite D, Weissman C. The new onset of atrial arrhythmias following major noncardiothoracic surgery is associated with increased mortality. Chest. 1998;114:462-8.
32. Whooley BP, Law S, Murthy SC, Alexandrou A, Wong J. Analysis of reduced death and complication rates after esophageal resection. Ann Surg. 2001;233:338-44.

33. Shimada M, Namai H, Morisaki H, Ochiai R, Takeda J, Fukushima K. [Preventive use of diltiazem to suppress supraventricular tachyarrhythmia in the patients after esophagectomy]. Masui. 1997;46(5):658-63.

\section{Targeted}

The Journal of Thoracic and Cardiovascular Surgery gives you two tables of contents.

The condensed table of contents tells you at a glance what topics and authors are presented each month. The expanded table of contents gives you a brief abstract of each article. You select only those articles of most interest to you for further reading. 